\title{
Multimodal influences on learning walks in desert ants (Cataglyphis fortis)
}

\author{
Jose Adrian Vega Vermehren ${ }^{1} \cdot$ Cornelia BuehImann ${ }^{2} \circledast$. Ana Sofia David Fernandes ${ }^{2} \cdot$ Paul Graham $^{2}$
}

Received: 20 April 2020 / Revised: 29 May 2020 / Accepted: 2 June 2020 / Published online: 15 June 2020

(c) The Author(s) 2020

\begin{abstract}
Ants are excellent navigators using multimodal information for navigation. To accurately localise the nest at the end of a foraging journey, visual cues, wind direction and also olfactory cues need to be learnt. Learning walks are performed at the start of an ant's foraging career or when the appearance of the nest surrounding has changed. We investigated here whether the structure of such learning walks in the desert ant Cataglyphis fortis takes into account wind direction in conjunction with the learning of new visual information. Ants learnt to travel back and forth between their nest and a feeder, and we then introduced a black cylinder near their nest to induce learning walks in regular foragers. By doing this across days with different wind directions, we were able to probe how ants balance different sensory modalities. We found that (1) the ants' outwards headings are influenced by the wind direction with their routes deflected such that they will arrive downwind of their target, (2) a novel object along the route induces learning walks in experienced ants and (3) the structure of learning walks is shaped by the wind direction rather than the position of the visual cue.
\end{abstract}

Keywords Learning walks $\cdot$ Ant navigation $\cdot$ Multimodal navigation $\cdot$ Visual cues $\cdot$ Wind

\section{Introduction}

Social insect foragers are expert navigators, using a combination of innate navigational strategies and learnt information from their environment (Knaden and Graham 2016). Early in their foraging life, ants have no information about their surroundings, and depend on idiothetic information which, via path integration (PI), is used to keep track of their approximate location relative to the nest (Mueller and Wehner 1988). PI allows ants to explore the world safely as navigationally useful visual (Zeil 2012; Collett et al. 2013) and olfactory (Steck 2012; Buehlmann et al. 2015) information

Jose Adrian Vega Vermehren and Cornelia Buehlmann are joint first author.

Electronic supplementary material The online version of this article (https://doi.org/10.1007/s00359-020-01431-9) contains supplementary material, which is available to authorized users.

Cornelia Buehlmann

cornelia.buehlmann@gmail.com

1 Westfaelische Hochschule, Bocholt, Germany

2 University of Sussex, School of Life Sciences, Brighton, UK is learnt to increase the reliability of localising the nest or following a foraging route.

The importance of learning environmental information for a forager is demonstrated by coordinated behavioural and physiological adaptations that mark the start of an ant's foraging career [reviewed in Roessler (2019)]. New foragers perform a specific set of exploratory walks that allow them to systematically inspect the surroundings of their nest [reviewed in Collett and Zeil (2018), and Zeil and Fleischmann (2019)]. During this early learning phase, ants do not search for food and only leave the nest for short periods, covering no more than a few centimetres before turning back (Wehner et al. 2004). The distance, time and path straightness then increase with each subsequent walk (Wehner et al. 2004; Fleischmann et al. 2016). Synchronised with this structured exploration, the brains of foragers show neuronal changes in the key brain areas associated with navigational systems and learning (Kuhn-Buehlmann and Wehner 2006; Stieb et al. 2010, 2012; Schmitt et al. 2016; Grob et al. 2017).

The structure of the learning walks of many species involves conspicuous turns and loops [Cataglyphis fortis: (Stieb et al. 2012; Fleischmann et al. 2016); Cataglyphis bicolor: (Wehner et al. 2004); Ocymyrmex robustior: 
(Mueller and Wehner 2010), Cataglyphis noda: (Fleischmann et al. 2017), Myrmecia croslandi: (Jayatilaka et al. 2018); Formica rufa: (Nicholson et al. 1999)]. Some species show fine-grained motor motifs such as voltes and pirouettes, which are small loops and nest-focussed inspections, respectively, on top of the coarse structure of loops and turns (Mueller and Wehner 2010; Fleischmann et al. 2017). Furthermore, the modulation of walking speed is also strongly correlated with the learning process of novel locations in $C$. fortis desert ants (Buehlmann et al. 2018). Thus, overall, we can identify a series of changes to walking patterns which constitute an active learning process during the learning walk behaviour in a variety of ant species.

Ants do not only perform learning walks when leaving the nest for the first time but engage in learning manoeuvres under other circumstances as well. Learning walks often occur on the first journey of each day, independent of the amount of previous experience the ant has had (Graham and Collett 2006) and also if the nest surroundings are changed significantly. For instance, if a cylinder is added near the ant's goal location, ants engage in learning walks to update their memories of the changed environment (Nicholson et al. 1999; Mueller and Wehner 2010).

The control of learning walks is inherently multimodal. Ants appear to use magnetic cues during early learning walks to control their orientation, with this early phase of learning walks giving an opportunity for ants to learn the configuration of celestial cues that are specific to the exact location and time of year (Fleischmann et al. 2018). However, it is less certain how learning walks are influenced by the need to learn multimodal information. Visual cues are clearly important, e.g., we see that the addition of extra visual information around the nest or a feeder location triggers new learning walks (Nicholson et al. 1999; Mueller and Wehner 2010), and those species that inhabit visually rich environments invest more in the fine control of motor motifs during learning walks (Fleischmann et al. 2017). What about the learning of information from other modalities? We know that during navigation, desert ants use information from olfaction (Buehlmann et al. 2012, 2014, 2015), wind direction (Wehner and Duelli 1971; Mueller and Wehner 2007), tactile cues (Seidl and Wehner 2006) and other sensory modalities. Wind direction is particularly interesting, as it can be used as a compass cue if it remains relatively constant over a period of time (Mueller and Wehner 2007) and it can also be a useful carrier of olfactory information (Wolf and Wehner 2000; Buehlmann et al. 2012, 2014, 2015; Steck 2012).

Here, we investigate whether the structure of learning walks of Cataglyphis fortis desert ants takes into account wind direction in conjunction with the learning of new visual information. We trained foragers to visit a regular feeder and then introduced a black cylinder near their nest; thus, learning walks were prompted in regular foragers. By doing this across days with different but constant wind directions, we were able to probe how ants balance the influence of different modalities.

\section{Materials and methods}

\section{Ants and field site}

Experiments were carried out between July and August of 2015 in the relatively featureless Tunisian salt pan near the village of Menzel Chaker (34.954897 N, 10.410396 E). Ten different nests of the desert ant species Cataglyphis fortis (Fig. 1a) were selected and each nest was only used once.

\section{Experimental procedures}

A circular arena ( $1 \mathrm{~m}$ in diameter) with a central, circular opening $(3 \mathrm{~cm})$ was placed over the nest entrance such that outgoing ants could only leave the nest by using the central hole and crossing the arena (Fig. 1b). Ants learnt to find a feeder that was placed $5 \mathrm{~m}$ away from the nest providing

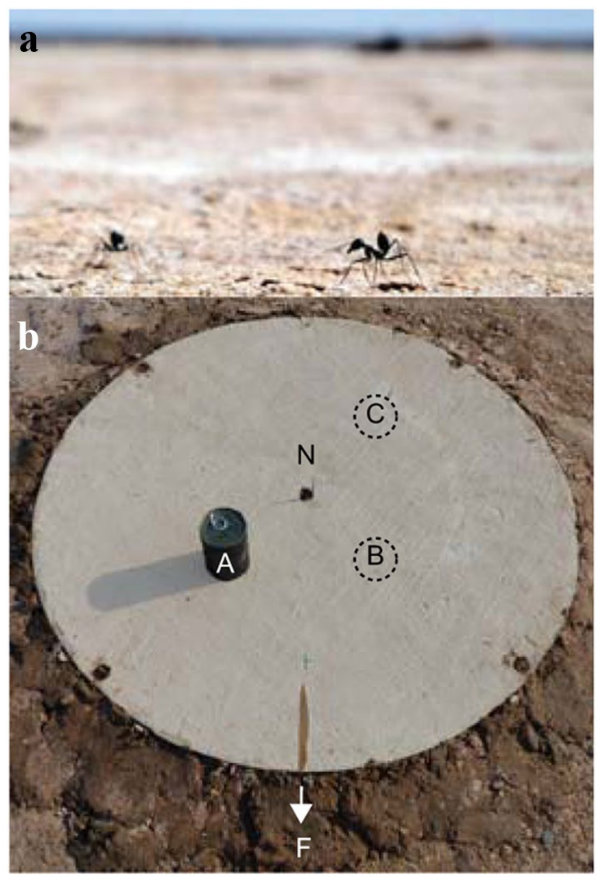

Fig. 1 Experimental setup. a Experiments were performed with the desert ant Cataglyphis fortis in the Tunisian salt pan. b A circular arena (1 $\mathrm{m}$ in diameter) with a central opening ( $3 \mathrm{~cm}$ in diameter) was placed on the top of the ants' nest $(\mathrm{N})$. Ants that could leave and enter the nest only through the central hole of the arena were trained to a feeder (F) $5 \mathrm{~m}$ away from the nest. In tests, a black cylinder $(7.5 \mathrm{~cm}$ in diameter, $11 \mathrm{~cm}$ high) was introduced $20 \mathrm{~cm}$ away from the nest at position A (Cyl-A), B (Cyl-B) or C (Cyl-C), respectively 
biscuit crumbs ad libitum for at least half a day. To control the movements of ants, fluon on the walls of the feeder stopped ants climbing out, unless a wooden stick was present as a bridge, which, during training, allowed ants to travel back and forth between the nest and the feeder.

The procedures for the control recordings were to (1) remove the wooden stick at the feeder to collect a batch of foragers, (2) put the stick back to let all ants return together back to the nest, (3) start recording departing outwards ants once most ants had entered the nest and (4) let them walk back and forth between the nest and feeder again. Similarly, the same procedures were followed for the recordings of the learning walks that were induced by adding a novel cylinder once most ants had entered the nest [between (2) and (3)]. Therefore, with a batch of regular foragers back in the nest, we could add the cylinder to induce learning walks on the ants' next emergence from the nest (Cylinder condition). The black cylinder $(7.5 \mathrm{~cm}$ in diameter, $11 \mathrm{~cm}$ high) was placed $20 \mathrm{~cm}$ away from the nest entrance in one of the 3 possible locations (Cyl-A, Cyl-B or Cyl-C) around the nest entrance (Fig. 1b). When time allowed, we collected the outwards ants in the feeder after their first exposure to the cylinder and recorded their subsequent inbound run in the presence of the novel cylinder.

Ants crossing the arena were recorded with a Panasonic DMC-F2200 high-speed camera (200 fps). A 5-cm-high stick with a string attached on the upper end was placed at the centre of the arena to record the wind direction throughout the experiments. To extract wind direction, movies were viewed in VLC movie player (version 3.0.3) and the direction of the string was categorised by eye into one of the 16 sectors (sector width $22.5^{\circ}$ ) every 240 frames $(1.2 \mathrm{~s}$ ). On all testing days, wind direction did not change more than $30^{\circ}$ during an experiment. In eight out of ten days, the prevailing wind direction was $55^{\circ}$ anticlockwise to the feeder direction $\left(+55^{\circ}\right)$. Because of the winds' consistency, these 8 days were grouped (Jul-07 to Jul-28). Mean wind direction on Jul-06 was $31^{\circ}$ clockwise $\left(-31^{\circ}\right)$ and on Aug-02 $125^{\circ}$ clockwise $\left(-125^{\circ}\right)$ to the feeder direction.

\section{Data processing and analysis}

The ants' paths were manually extracted from videos at 20 fps using Graph Click (Arizona-Software, version 3.0). Tracking started when the last shadow cast by the experimenter was out of sight. Every ant leaving the nest entrance and venturing more than $2 \mathrm{~cm}$ away from the nest was tracked. Ants interacting with other ants were tracked as far as possible. If no distinction between two interacting ants was possible, the files were labelled, and each ant was matched with one of the departing ants. Ants disappearing behind the cylinder (from the camera's perspective) were followed to the edge and then matched with a departing ant (from behind the cylinder) as accurately as possible. To ensure that all inwards ants came from our feeder, we only considered homing ants that entered the arena from the feeder direction $\pm 90^{\circ}$.

Calibration marks on the arena were used for calibration and digitised paths were smoothed with a mean filter and a window size of three frames. Path straightness was calculated as the quotient of the beeline, i.e., the euclidean distance between the first and last point of a path, and the actual path length (Index of straightness, IS), with paths containing gaps shifted to close gaps. For more detailed path analysis, paths were divided into chunks. The length of these chunks was set to $2 \mathrm{~cm}$ and the direction of each chunk and the ant's speed in that chunk was calculated. Chunks were considered to be aligned to the nest when the angular difference to the nest was smaller than $22.5^{\circ}$. To find chunks with turns, we looked for chunks where the distance from the nest entrance to a chunk had reduced by more than $1 \mathrm{~cm}$ (half a chunk) since the previous chunk. For the analysis of walking speed, a threshold of $0.06 \mathrm{~m} / \mathrm{s}$ was set to identify path segments with low walking speed that may indicate learning (Buehlmann et al. 2018).

All data were analysed and plotted using Matlab R2017b. Statistical analysis was performed using the Statistics and Machine Learning Toolbox, as well as the Circular Statistics Toolbox (Berens 2009). Furthermore, additional circular statistics (Batschelet 1981) were run using Oriana 4.02 (Kovach computing services) and PAST (Hammer et al. 2001).

\section{Results}

\section{Ants' outwards headings are influenced by the direction of the wind}

The Control ants' heading directions on days with the prevailing wind ( $+55^{\circ}$; Jul-07 to Jul-28) and on days with wind $31^{\circ}$ negative to the feeder direction $\left(-31^{\circ}\right)$ could be predicted by both the feeder and the wind direction at three different distances from the nest entrance (ants with wind $+55^{\circ}: V$ test, $n=216$ ants, $r_{1}=10 \mathrm{~cm}, r_{2}=25 \mathrm{~cm}$, $r_{3}=45 \mathrm{~cm}$, feeder: $p_{1-3}<0.001$, mean wind: $p_{1-3}<0.001$; ants with wind $-31^{\circ}: V$ test, $n=23$ ants, feeder: $p_{1-3}<0.001$, mean wind: $\left.p_{1-3}<0.001\right)$. The wind on Aug-02 blew in a direction almost opposite to the feeder $\left(-125^{\circ}\right.$ to the feeder direction). Here, the ants' heading direction could be predicted by the feeder but not the wind direction ( $V$ test, $n=36$ ants, feeder: $p_{1-3}<0.01$, mean wind: $p_{1-3}>0.05$ ). Moreover, the spread in the ants' heading directions was significantly higher than in the other two wind conditions $(k$ test with Bonferroni correction, $-30^{\circ}$ vs. $+55^{\circ}, p_{1-3}>0.05 ;-30^{\circ}$ vs. $-125^{\circ}, p_{1-3}<0.01 ;+55^{\circ}$ vs. $\left.-125^{\circ}, p_{1-3}<0.001\right)$. 
Taken together, wind direction had an influence on the ants' heading directions, and when in a directional conflict with the feeder direction, the ants' directional scatter increased significantly.

\section{Novel object along the route induces learning walks in experienced ants}

Experienced ants navigating to the feeder were introduced to a black cylinder placed at different positions relative to the nest (Fig. 1b). To analyse the impact of the different cylinder positions on the ants' paths but discard the influence of the different wind directions, only experiments from days with the prevailing wind direction were analysed here $\left(+55^{\circ}\right.$; Jul-07 to Jul-28). In general, the ants' headings were directed (Rayleigh test, all $p<0.001$ ) and the mean heading direction in the Control and Cylinder condition did not differ from each other (ants' headings at $r_{1}=10 \mathrm{~cm}, r_{2}=25 \mathrm{~cm}, r_{3}=45 \mathrm{~cm}$; Control vs. Cylinder, Watson Williams tests, $p<0.05$ for $r_{1}$ in Cyl-A, everything else $p>0.05$; see Fig. 2). However, the spread of heading directions differed significantly between Control and Cylinder conditions in cylinder position $\mathrm{A}$ and $\mathrm{B}$, but not in
$\mathrm{C}$ (Control vs. Cylinder, $k$ tests, $p<0.05$ for all three distances in Cyl-A and Cyl-B, $p>0.05$ for all three distances in Cyl-C; Fig. 2). Similarly, overall path straightness was significantly decreased in cylinder positions A and B but not cylinder position $\mathrm{C}$ (Index of straightness: Control vs. Cylinder, Mann Whitney $U$ test, $p<0.001$ in Cyl-A, $p<0.05$ in Cyl-B, $p>0.05$ in Cyl-C). Average walking speed did not change in any of the conditions (Control vs. Cylinder, Mann-Whitney $U$ test, $p>0.05$ for all three cylinder positions). Also, in all groups, some ants returned back to the nest without leaving the arena; however, the proportion of ants returning to the nest did not differ between the Control and Cylinder conditions (Control vs. Cylinder, Chi-squared test, $p>0.05$ for all three cylinder positions). In summary, the presence of a novel cylinder in the nest vicinity induced learning walks when it was placed along the learnt route to the feeder (position $\mathrm{A}$ and B) but not when it was placed at position C.

When time allowed, we additionally recorded the ants' first nest return after having added the unfamiliar cylinder (Fig. S1). Similar to what we have seen in outwards ants, we observed in inwards ants that the overall change in paths was bigger for cylinder position A than C (Fig. S1). However,
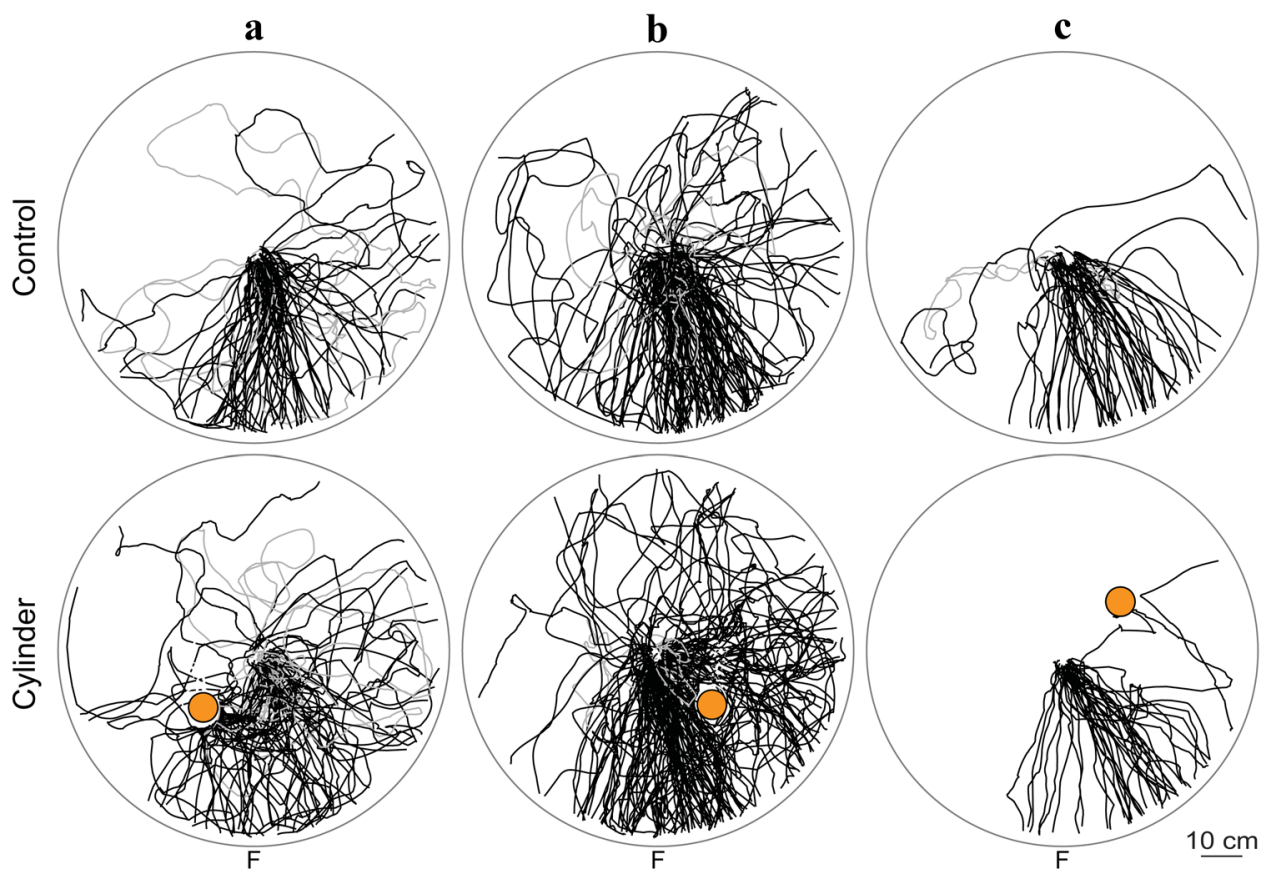

Fig. 2 Outwards ants heading to the feeder when familiar with the nest surrounding (top) and when a novel cylinder is added (bottom). Trajectories from individual ants leaving the nest (located at the centre of the circular arena) and entirely crossing the arena ( $1 \mathrm{~m}$ in diameter) are shown as black lines. Ants returning to the nest without leaving the arena are shown in grey. Dashed lines connect gaps in paths. Cylinder is shown in orange. Ants from different nests, but with the same cylinder positions (Cyl-A, Cyl-B or Cyl-C) are grouped together. Prevailing wind on these days was $55^{\circ}$ anticlockwise of the feeder direction $\left(+55^{\circ}\right)$. a Jul-13, Jul-26, Jul-28; Control, $n=71$ ants, nest returns (ants not entirely crossing the arena but returning to the nest): 5 out of 71 ants; Cylinder, $n=79$ ants, nest returns: 12 out of 79 ants. b Jul-07, Jul-14, Jul-16; Control, $n=129$ ants, nest returns: 8 out of 129; Cylinder, $n=158$ ants, nest returns: 15 out of 158 ants. c Jul-17, Jul-27; Control, $n=53$ ants, nest returns: 2 out of 53 ants; Cylinder, $n=39$ ants, nest returns: 0 out of 39 ants 
control ants from the cylinder position B were noisier than usual and, thus, made the comparison difficult.

\section{The novel object induces sensorimotor motifs characteristic of learning}

In addition to general path characteristics described above, we looked for path segments with decreased walking speed, increased turning and alignment with the nest. The proportion of ants with path segments of low walking speed did not differ between Control and Cylinder conditions (Control vs. Cylinder, Chi-square test, all $p>0.05$ ). However, ants more often performed turns and aligned themselves towards the nest when the cylinder was added in position $\mathrm{A}$ with the prevailing wind (Turns: Control vs. Cylinder, Chi-square test, $p<0.001$; nest alignments: Control vs. Cylinder, Chi-square test, $p<0.01)$ and position B with an atypical wind direction (Turns: Control vs. Cylinder, Chi-square test, $p<0.01$; nest alignments: Control vs. Cylinder, Chi-square test, $p<0.01$ ), but not in position A with an atypical wind direction (Turns: Control vs. Cylinder, Chi-square test, $p>0.05$; Nest alignments: Control vs. Cylinder, Chi-square test, $p>0.05)$ and position B with the prevailing wind direction
(Turns: Control vs. Cylinder, Chi-square test, $p>0.05$; Nest alignments: Control vs. Cylinder, Chi-square test, $p>0.05$ ). Figures 3, 4 and 5 show the wind directions for the above conditions and we can see that there is a significant increase in the sensorimotor motifs associated with learning in those conditions where the wind biases route directions such that ants run close to the cylinder.

\section{Structure of learning walks is shaped by the wind direction}

To analyse how the fine structure of learning walks was influenced by the direction of the wind, we analysed in fine detail the ants' walking speed (Fig. 3), turning behaviour (Fig. 4) and facing direction (Fig. 5) for cylinder positions A and $\mathrm{B}$. To do so, we compared ants from conditions with the same cylinder position but different wind directions. Because the data from some groups was too scattered (see Rayleigh tests with $p>0.05$ in Figs. 3, 4 and 5), we focused on cylinder position $\mathrm{B}$ (wind $+55^{\circ}$ to the feeder vs. wind direction $-31^{\circ}$ to the feeder). We found that the wind direction had a significant influence on the positional distribution of path sections with low walking speed, turns and nest alignments,

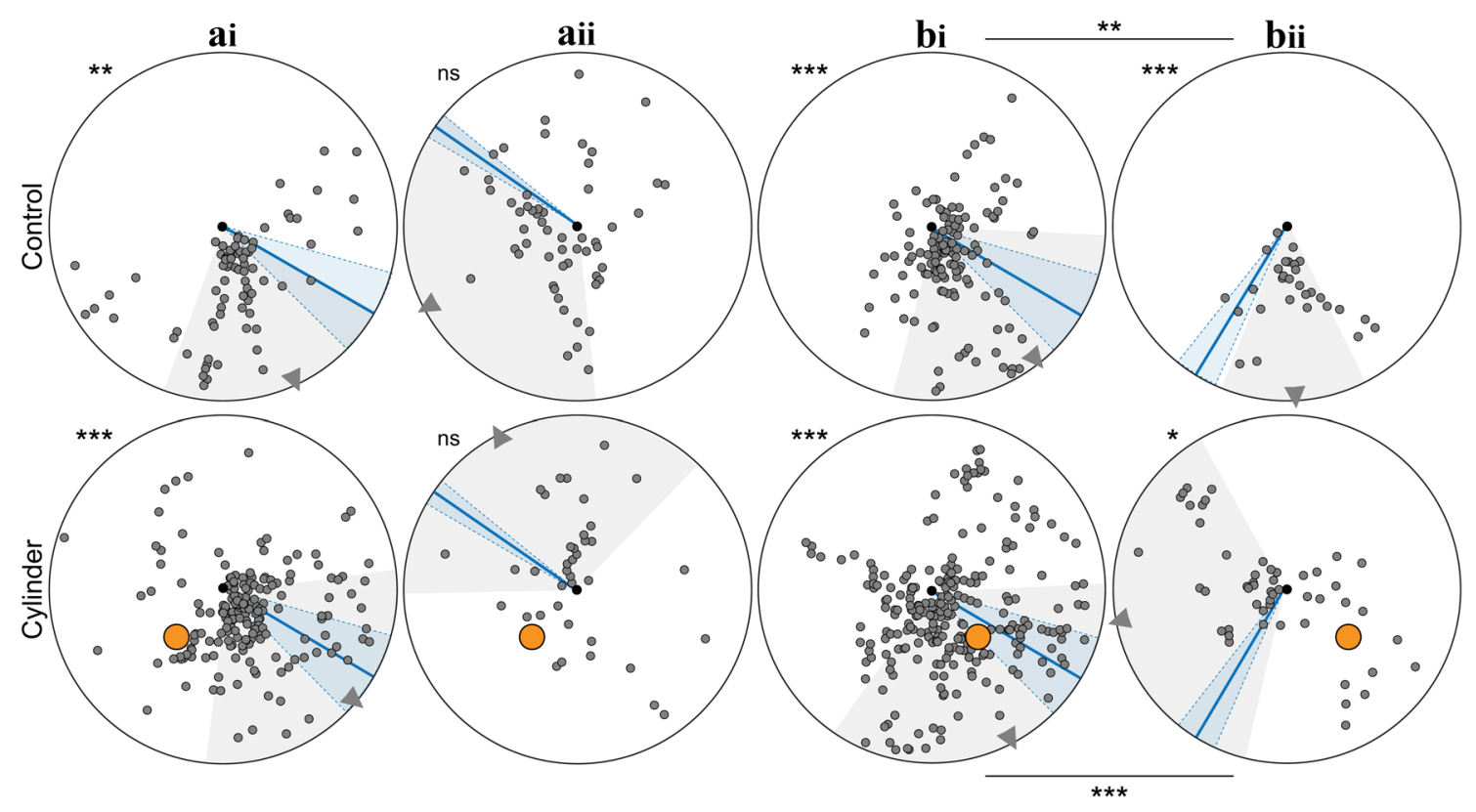

Fig. 3 Path segments with low walking speed when ants are familiar with the nest surrounding (top) and when a novel cylinder is added (bottom). Path segments ( $2 \mathrm{~cm}$ chunks) where ants walked slower than $0.06 \mathrm{~m} / \mathrm{s}$ are shown as small grey circles. Nest, black dot in centre of arena. Cylinder is shown in orange. Ants from different nests, but with the same cylinder position (Cyl-A or Cyl-B) are grouped together. Wind direction: mean with standard deviation (blue line and shadow). Direction of selected path chunks: mean with standard deviation (grey triangle and shadow). For the statistics, the circular mean of the angular positions (relative to the nest) of the path segments with low walking speed was calculated for each ant. Directionality of data was tested using the Rayleigh tests: asterisks outside of circular arena, $p<0.05(*), p<0.01(* *), p<0.001(* * *)$, not significant (ns). If the data was directed, Watson Williams tests were used to do pairwise comparisons of a specific cylinder position with different wind directions. bi vs. bii, Control $p<0.01$ (**), Cylinder $p<0.001$ (***). ai: wind $+55^{\circ}$ to the feeder direction (prevailing wind), aii: wind $-125^{\circ}$ to the feeder direction, bi: wind $+55^{\circ}$ to the feeder direction (prevailing wind), bii: wind $-31^{\circ}$ to the feeder direction 


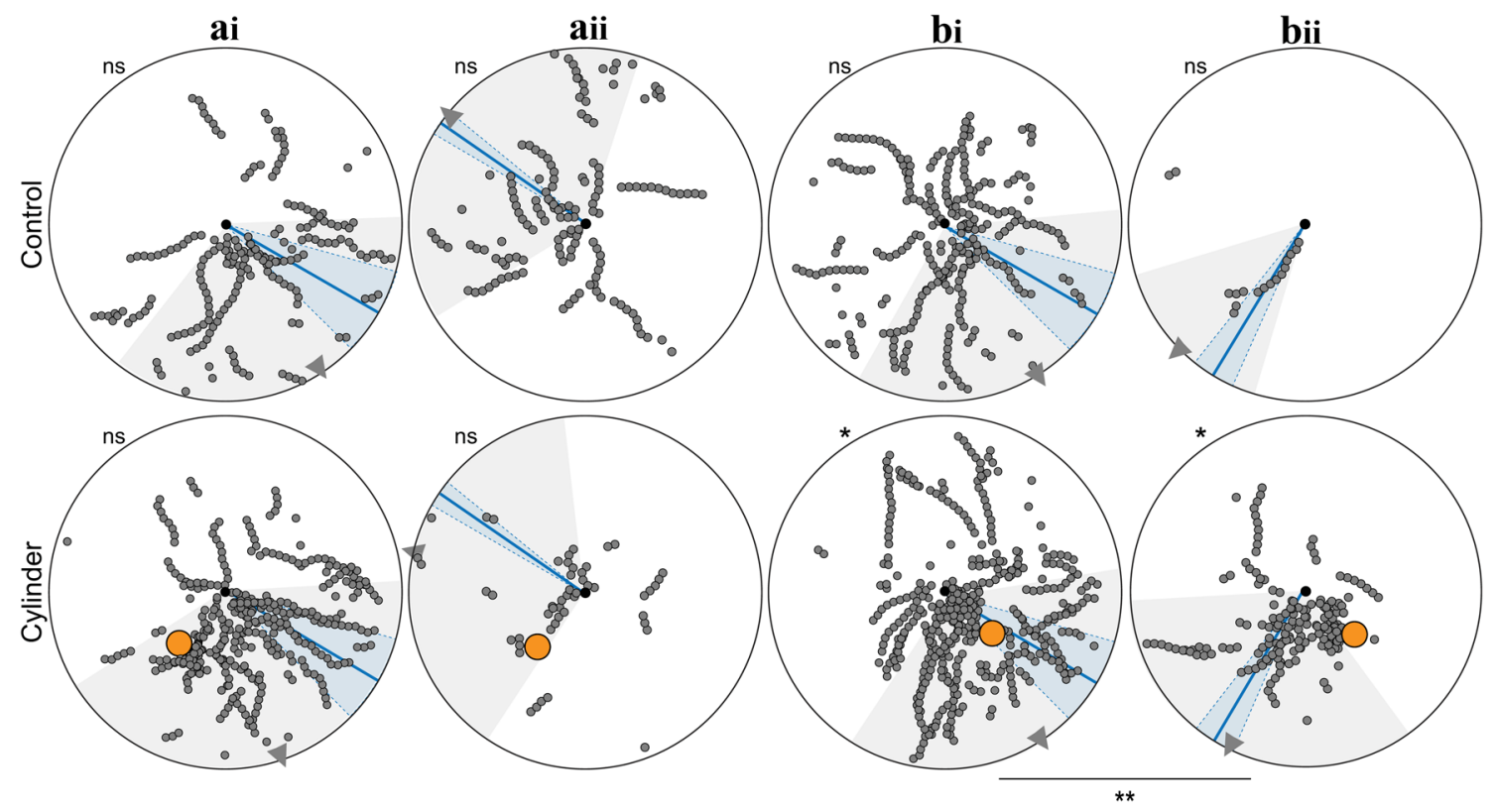

Fig. 4 Path segments with turns when ants are familiar with the nest surrounding (top) and when a novel cylinder is added (bottom). Path chunks where ants turned are shown as small grey circles. Nest, black dot in centre of arena. Cylinder is shown in orange. Ants from different nests, but with the same cylinder position (Cyl-A or Cyl-B) are grouped together. Wind direction: mean with standard deviation (blue line and shadow). Direction of selected path chunks: mean with standard deviation (grey triangle and shadow). For the statistics, the circular mean of the angular positions (relative to the nest) of the path

respectively. The spatial distribution of the path segments with low walking speed (bi vs. bii in Fig. 3; Watson Williams test, Control $p<0.01$. Cylinder $p<0.001$ ), turns (bi vs. bii in Fig. 4; Watson Williams test, Cylinder, $p<0.01)$ and also nest alignments (bi vs. bii in Fig. 5; Watson Williams test, Cylinder, $p<0.05$ ) was significantly influenced by the direction of the wind. Hence, learning walks are adapted to take into account the current directionality of wind.

\section{Discussion}

Our aim was to investigate whether the structure of learning walks of Cataglyphis fortis desert ants is influenced by the wind direction in conjunction with the learning of new visual information. We trained foragers to visit a regular feeder and then introduced a black cylinder near their nest; thus, learning walks (or re-learning walks) were provoked in regular foragers. By doing this across days with different but constant wind directions, we were able to probe how ants compromise across information derived from different modalities.

It has been demonstrated previously that wind direction plays a role in the approach strategies of ants to unfamiliar segments with turns was calculated for each ant. Directionality of data was tested using the Rayleigh tests: asterisks outside of circular arena, $p<0.05(*), p<0.01(* *), p<0.001$ (***), not significant (ns). If the data was directed, Watson Williams tests were used to do pairwise comparisons of a specific cylinder position with different wind directions. bi vs. bii, Cylinder $p<0.01(* *)$. ai: wind $+55^{\circ}$ to the feeder direction (prevailing wind), aii: wind $-125^{\circ}$ to the feeder direction, bi: wind $+55^{\circ}$ to the feeder direction (prevailing wind), bii: wind $-31^{\circ}$ to the feeder direction

(Buehlmann et al. 2014) and familiar feeding locations (Wolf and Wehner 2000, 2005) as well as to the nest (Buehlmann et al. 2012). In all these scenarios, the final approach is into the wind, which maximises the potential to use olfactory information. Similarly, the paths from bumble bees leaving or approaching the nest were shown to be shaped by wind direction (de Ibarra et al. 2009). In agreement with these previous observations, we see that the departure directions of our ants are influenced by the current wind direction, with forager routes deflected in such a way that ants will arrive downwind of their target. Additionally, by biasing the departure direction of ants' foraging routes, the wind direction indirectly acts to determine the locations and structure of the provoked learning walks. From the perspective of an individual forager upon leaving the nest, the initial foraging direction is chosen as an integration of the remembered food direction, and the direction of the wind [as in (Wolf and Wehner 2005)]. If that path direction means that the ant's path runs close by the newly installed landmark (cylinder positions A and B, vs. cylinder position $C$ ), then we see an upregulation in the sensorimotor motifs associated with learning. However, the landmark does not act as a direct driver of the precise locations of the learning behaviours. For instance, if the 


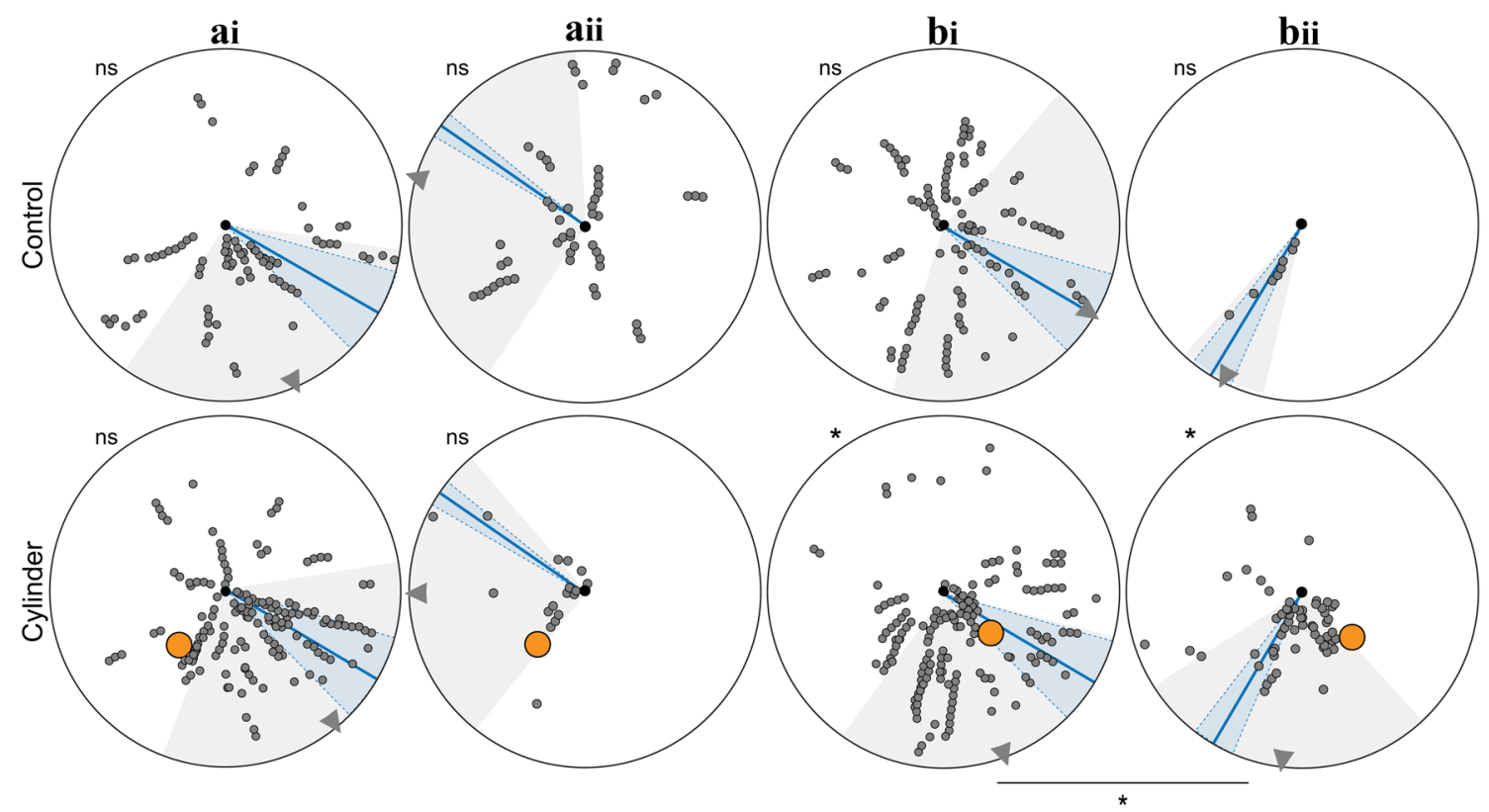

Fig. 5 Nest alignments when familiar with the nest surrounding (top) and when a novel cylinder is added (bottom). Path segments that were aligned to the nest are shown as small grey circles. Nest, black dot in centre of arena. Cylinder is shown in orange. Ants from different nests, but with same cylinder positions (Cyl-A or Cyl-B) are grouped together. Wind direction: mean with standard deviation (blue line and shadow). Direction of selected path chunks: mean with standard deviation (grey triangle and shadow). For the statistics, the circular mean of the angular positions (relative to the nest) of the nest align- ments was calculated for each ant. Directionality of data was tested using the Rayleigh tests: asterisks outside of circular arena, $p<0.05$ $(*), p<0.01(* *), p<0.001(* * *)$, not significant (ns). If the data was directed, Watson Williams tests were used to do pairwise comparisons of a specific cylinder position with different wind directions. bi vs. bii, Cylinder $p<0.05\left(^{*}\right)$. ai: wind $+55^{\circ}$ to the feeder direction (prevailing wind), aii: wind $-125^{\circ}$ to the feeder direction, bi: wind $+55^{\circ}$ to the feeder direction (prevailing wind), bii: wind $-31^{\circ}$ to the feeder direction landmark position had a definitive influence on the structure of the learning walks, inspections following the landmark being added to positions $\mathrm{A}$ and $\mathrm{B}$ would be mirrorsymmetric to each other, given the same wind conditions.

To indicate those portions of routes that might be indicative of 're-learning walks', we looked for slowing (Fig. 3), turning (Fig. 4) and nest inspections (Fig. 5). We know that across a range of ant species, there are significant differences in the sensorimotor motifs that signify learning walks (Fleischmann et al. 2017; Zeil and Fleischmann 2019). The sensory ecology of an ant species seems to be the major determinant of the style of their learning walks, although most of our understanding comes from examples of ant species in habitats with different amounts of visual clutter. Our results suggest that other sensory modalities also need to be considered when cataloguing the relationship between learning walks and habitat. It might be that Cataglyphis fortis foragers take advantage of the relatively constant wind direction to enhance the utility of learning walks, and whilst they are clearly influenced by the addition of the landmark, they may place less weight on the precise learning of the visual cue. Further experiments, especially with brand new foragers, would allow us to investigate how individual species give appropriate weight to wind information relative to the information from visual clutter in their habitat. Similarly, it would be interesting to investigate the role of the wind in experienced ants inhabiting environments of different visual clutter.

To implement these experiments, we needed to record ants on days with a range of wind conditions; however, it is true that for this habitat, there was a prevailing wind direction which was seen on most days. The influence of this being that most foraging paths had a characteristic deflection, and so over a day-to-day period, the habitual routes of foragers would have this shape. This raises the question of to what extent, wind information is incorporated into the route memories of regular foragers. Does the wind set a route direction, but then route guidance information is learnt independent of the wind? Or, is wind information part of the multimodal sensory information used to guide the habitual routes? The first of these options is a pattern of route learning that is seen in other modalities (Collett et al. 2003) when a particular cue determines (or scaffolds) the shape of the learnt habitual route, but then, for experienced foragers, the habitual route can be accurately navigated without the original scaffold. This pattern of learning can be seen when visual cues (Graham et al. 2003), path integration (Collett et al. 2001) or pheromone trails (Harrison et al. 1989) are used 
to determine an initial path shape, which is still maintained in experienced foragers, even when the original scaffold is removed. In these experiments, we did not have marked ants of known identity or experience; therefore, it is impossible to determine the ontogeny of route development. However, on days when the wind direction showed a strong change away from the prevailing direction, we do see significant disturbance to outwards paths. Assuming that a significant proportion of the disturbed ants were experienced individuals, it suggests that wind cues are part of the multimodal suite of cues used to guide routes (Buehlmann et al. 2020), rather than simply a temporary scaffold during learning.

Acknowledgements This project was funded by the people programme (Marie Curie Actions) of the European Union's Seventh Framework Programme (FP7/2007-2013, under REA grant agreement no. PIEFGA-2013-624765) to CB. PG is additionally funded by a BBSRC grant $\mathrm{BB} / \mathrm{R} 005036 / 1$ and EPSRC grant EP/P006094/1 and CB is additionally funded by a BBSRC grant BB/R005036/1. JAVV was hosted within the Brains on Board project.

Data availability Data are available from the University of Sussex research repository: https://doi.org/10.25377/sussex.12462152.

\section{Compliance with ethical standards}

Conflict of interest The authors declare that they have no conflict of interest.

Ethical approval All applicable international, national, and institutional guidelines for the care and use of animals were followed.

Open Access This article is licensed under a Creative Commons Attribution 4.0 International License, which permits use, sharing, adaptation, distribution and reproduction in any medium or format, as long as you give appropriate credit to the original author(s) and the source, provide a link to the Creative Commons licence, and indicate if changes were made. The images or other third party material in this article are included in the article's Creative Commons licence, unless indicated otherwise in a credit line to the material. If material is not included in the article's Creative Commons licence and your intended use is not permitted by statutory regulation or exceeds the permitted use, you will need to obtain permission directly from the copyright holder. To view a copy of this licence, visit http://creativecommons.org/licenses/by/4.0/.

\section{References}

Batschelet E (1981) Circular statistics in biology. Academic Press, New York

Berens P (2009) CircStat: aA MATLAB toolbox for circular statistics. J Stat Softw. https://doi.org/10.18637/jss.v031.i10

Buehlmann C, Hansson BS, Knaden M (2012) Path integration controls nest-plume following in desert ants. Curr Biol 22:645-649. https ://doi.org/10.1016/j.cub.2012.02.029

Buehlmann C, Graham P, Hansson BS, Knaden M (2014) Desert ants locate food by combining high sensitivity to food odors with extensive crosswind runs. Curr Biol 24:960-964. https://doi. org/10.1016/j.cub.2014.02.056
Buehlmann C, Graham P, Hansson BS, Knaden M (2015) Desert ants use olfactory scenes for navigation. Anim Behav 106:99-105. https://doi.org/10.1016/j.anbehav.2015.04.029

Buehlmann C, Fernandes ASD, Graham P (2018) The interaction of path integration and terrestrial visual cues in navigating desert ants: what can we learn from path characteristics? J Exp Biol. https://doi.org/10.1242/jeb.167304

Buehlmann C, Mangan M, Graham P (2020) Multimodal interactions in insect navigation. Anim Cogn. https://doi.org/10.1007/s1007 $1-020-01383-2$

Collett TS, Zeil J (2018) Insect learning flights and walks. Curr Biol 28:R984-R988. https://doi.org/10.1016/j.cub.2018.04.050

Collett TS, Collett M, Wehner R (2001) The guidance of desert ants by extended landmarks. J Exp Biol 204:1635-1639

Collett TS, Graham P, Durier V (2003) Route learning by insects. Curr Opin Neurobiol 13:718-725. https://doi.org/10.1016/j. conb.2003.10.004

Collett M, Chittka L, Collett T (2013) Spatial memory in insect navigation. Curr Biol 23:R789-R800. https://doi.org/10.1016/j. cub.2013.07.020

de Ibarra NH, Philippides A, Riabinina O, Collett TS (2009) Preferred viewing directions of bumblebees (Bombus terrestris L.) when learning and approaching their nest site. J Exp Biol 212:3193-3204. https://doi.org/10.1242/jeb.029751

Fleischmann PN, Christian M, Mueller VL, Roessler W, Wehner R (2016) Ontogeny of learning walks and the acquisition of landmark information in desert ants, Cataglyphis fortis. J Exp Biol 219:3137-3145. https://doi.org/10.1242/jeb.140459

Fleischmann PN, Grob R, Wehner R, Roessler W (2017) Speciesspecific differences in the fine structure of learning walk elements in Cataglyphis ants. J Exp Biol 220:2426-2435. https:// doi.org/10.1242/jeb.158147

Fleischmann PN, Grob R, Muller VL, Wehner R, Roessler W (2018) The geomagnetic field is a compass cue in Cataglyphis ant navigation. Curr Biol 28:1440-1444. https://doi.org/10.1016/j. cub.2018.03.043

Graham P, Collett TS (2006) Bi-directional route learning in wood ants. J Exp Biol 209:3677-3684. https://doi.org/10.1242/ jeb.02414

Graham P, Fauria K, Collett TS (2003) The influence of beaconaiming on the routes of wood ants. J Exp Biol 206:535-541. https://doi.org/10.1242/jeb.00115

Grob R, Fleischmann PN, Grubel K, Wehner R, Roessler W (2017) The role of celestial compass information in Cataglyphis ants during learning walks and for neuroplasticity in the central complex and mushroom bodies. Front Behav Neurosci. https://doi. org/10.3389/fnbeh.2017.00226

Hammer O, Harper DAT, Ryan PD (2001) PAST: Paleontological statistics software package for education and data analysis. Palaeontol Electron 4:9

Harrison JF, Fewell JH, Stiller TM, Breed MD (1989) Effects of experience on use of orientation cues in the giant tropical ant. Anim Behav 37:869-871. https://doi.org/10.1016/00033472(89)90076-6

Jayatilaka P, Murray T, Narendra A, Zeil J (2018) The choreography of learning walks in the Australian jack jumper ant Myrmecia croslandi. J Exp Biol. https://doi.org/10.1242/jeb.185306

Knaden M, Graham P (2016) The sensory ecology of ant navigation: from natural environments to neural mechanisms. Annu Rev Entomol 61:63-76. https://doi.org/10.1146/annurev-ento-01071 5-023703

Kuhn-Buehlmann S, Wehner R (2006) Age-dependent and taskrelated volume changes in the mushroom bodies of visually guided desert ants, Cataglyphis bicolor. J Neurobiol 66:511521. https://doi.org/10.1002/neu.20235 
Mueller M, Wehner R (1988) Path integration in desert ants, Cataglyphis fortis. Proc Natl Acad Sci USA 85:5287-5290. https ://doi.org/10.1098/rspb.2004.2749

Mueller M, Wehner R (2007) Wind and sky as compass cues in desert ant navigation. Naturwissenschaften 94:589-594. https ://doi.org/10.1007/s00114-007-0232-4

Mueller M, Wehner R (2010) Path integration provides a scaffold for landmark learning in desert ants. Curr Biol 20:1368-1371. https://doi.org/10.1016/j.cub.2010.06.035

Nicholson DJ, Judd SPD, Cartwright BA, Collett TS (1999) Learning walks and landmark guidance in wood ants (Formica rufa). J Exp Biol 202:1831-1838

Roessler W (2019) Neuroplasticity in desert ants (Hymenoptera: Formicidae)-importance for the ontogeny of navigation. Myrmecol News 29:1-20. https://doi.org/10.25849/myrmecol. news_029:001

Schmitt F, Stieb SM, Wehner R, Roessler W (2016) Experience-related reorganization of giant synapses in the lateral complex: potential role in plasticity of the sky-compass pathway in the desert ant Cataglyphis fortis. Dev Neurobiol 76:390-404. https://doi. org/10.1002/dneu.22322

Seidl T, Wehner R (2006) Visual and tactile learning of ground structures in desert ants. J Exp Biol 209:3336-3344. https://doi. org/10.1242/jeb.02364

Steck K (2012) Just follow your nose: homing by olfactory cues in ants. Curr Opin Neurobiol 22:231-235. https://doi.org/10.1016/j. conb.2011.10.011

Stieb SM, Muenz TS, Wehner R, Roessler W (2010) Visual experience and age affect synaptic organization in the mushroom bodies of the desert ant Cataglyphis fortis. Dev Neurobiol 70:408-423. https ://doi.org/10.1002/dneu.20785

Stieb SM, Hellwig A, Wehner R, Roessler W (2012) Visual experience affects both behavioral and neuronal aspects in the individual life history of the desert ant Cataglyphis fortis. Dev Neurobiol 72:729-742. https://doi.org/10.1002/dneu.20982

Wehner R, Duelli P (1971) Spatial orientation of desert ants, Cataglyphis bicolor, before sunrise and after sunset. Experientia 27:13641366. https://doi.org/10.1007/BF02136743

Wehner R, Meier C, Zollikofer C (2004) The ontogeny of foraging behaviour in desert ants, Cataglyphis bicolor. Ecol Entomol 29:240-250. https://doi.org/10.1111/j.0307-6946.2004.00591.x

Wolf H, Wehner R (2000) Pinpointing food sources: olfactory and anemotactic orientation in desert ants, Cataglyphis fortis. J Exp Biol 203:857-868

Wolf H, Wehner R (2005) Desert ants compensate for navigation uncertainty. J Exp Biol 208:4223-4230. https://doi.org/10.1242/ jeb.01905

Zeil J (2012) Visual homing: an insect perspective. Curr Opin Neurobiol 22:285-293. https://doi.org/10.1016/j.conb.2011.12.008

Zeil J, Fleischmann PN (2019) The learning walks of ants (Hymenoptera: Formicidae). Myrmecol News 29:93-110. https://doi. org/10.25849/myrmecol.news_029:093

Publisher's Note Springer Nature remains neutral with regard to jurisdictional claims in published maps and institutional affiliations. 